\title{
Influence of manganese doping on elastic and structural properties of silica borotellurite glass
}

\begin{abstract}
The quaternary glass system tellurium dioxide, boron trioxide, silicon dioxide and manganese dioxide where $\mathrm{x}=0.00$ to $0.05 \mathrm{M}$ fraction has been successfully prepared via conventional melt and quench-casting technique. The density was determined by Archimedes method with distilled water as buoyant liquid. The results showed that the density decreases and molar volume increases with respect to manganese concentration. FTIR spectral studies suggest that glass network is mainly built up of boron trioxide, tetrahedral boron, tellurium trioxide, trioxotellurate and silicon dioxide structural units. The ultrasonic velocities and elastic moduli were recorded using a pulse-echo method at frequency $5 \mathrm{MHz}$ at room temperature. Some other physical properties such as Debye temperature, softening temperature, microhardness, Poisson's ratio, fugacity, fractal bond connectivity and Cauchy relation were determined. Results indicate that these parameters depend upon the composition of the glass system and the dopant concentration, $\mathrm{MnO} 2$ inside the glass system.
\end{abstract}

Keyword: Elastic moduli; Ultrasonic velocities; Debye temperature; FTIR 